\title{
ANOTACIONES AL TEXTO DEL LIBRO II DE TITO LIVIO
}

Some fifty passages of the book II of Tite-Live, for which the author proposes lectures different from those of the last editions, specially the excellent one of $\mathbf{R}$. $\mathbf{M}$. Ogilvie (Oxford Classical Texts, 1974), are discussed here. So the author tries to justify the text adopted in his edition of the "Colección Hispánica de Autores Griegos y Latinos" (Madrid 1987). Only four of the here offered lectures are new conjectures. In all the others, whether the supposed lecture of the Nicomachean archetype or those of former editors are recovered. The prevailing criteria applied in this paper are the stemmatic one and the usus scribendi auctoris. A similar paper concerning the book I of the Roman historian was published in EMERITA 42, 1974, pp. 343-373, and 43,1975 , pp. 1-24.

Mi edición de los libros I y II de Tito Livio en la «Colección Hispánica de Autores Griegos y Latinos» ha supuesto una revisión del texto, para la que me ha servido de referencia básica el publicado en la serie de Oxford (1974) por R. M. Ogilvie, el inteligente y muy erudito especialista británico cuya prematura desaparición fue tan lamentada en los ambientes filológicos y del «anticuarismo» romano.

Aunque los trabajos de Ogilvie $^{\prime}$ han representado una culminación de los estudios sobre la tradición de la I década de Livio y sobre la interpretación y el texto de los libros $\mathrm{I}-\mathrm{V}$, mi edición revisada se aparta en cierto número de lugares de la del inglés. En algunas ocasiones he pensado que podia ajustarme más literalmente a las lecturas de los manuscritos; a veces me han convencido más las opciones de otros editores que la de Ogilvie y he vuelto a ellas; en ciertos casos, en fin, he ofrecido una conjetura propia, elaborada a partir de alguna que había sido propuesta con anterioridad.

\footnotetext{
I R. M. Ogilvie, "The manuscript tradition of Livy's First Decade», $C Q 7$, 1957. pp. 68-81; Id., "Livius Resartus», $C Q 9$ 9, 1959, pp. 269-284; Id., A Commentary on Livy. Books 1-5, Oxford 1965 (citado: Ogilvie, A Comm.); T. Liui Ab urbe condita Libri I-V, ed. R. M. Ogilvie, OCT, Oxonii 1974.
} 
Las notas reunidas en este artículo pretenden dar cuenta de lugares en que mi texto del libro II difiere del oxoniense. Son una continuación del trabajo relativamente extenso publicado en esta misma revista hace una docena de años y que se ceñia al libro $I^{2}$.

Aquel estudio coincidió en el tiempo con la edición de Ogilvie, pero contaba con las lecturas que el británico había avanzado en su rico y razonado comentario. Después de mi artículo y de su edición, el profesor Ogilvie y yo nos cruzamos unas cartas con algunas de nuestras respectivas apreciaciones. Resultó para mí halagüeño que él me dijera que, tras estudiar mis notas, habría modificado el texto de su libro I en casi una docena de pasajes. Yo, tras leer su correspondencia, también tendría que retirar mis sugerencias en otros tantos casos, lo cual se refleja en que en la edición no he mantenido algunas de mis propuestas de entonces. Pero entiendo que con mis "Anotaciones» de los años 74 y 75 y otras cosas publicadas después por diversos estudiosos acerca de ese libro I, mi revisión crítica de él está suficientemente explicada.

A continuación presento una relación de los pasajes del libro II en que mi texto de la "Colección Hispánica» discrepa del de OCT. Este trabajo de ahora ha sido concebido como una continuación del anterior y como un apoyo crítico a mi edición. A efectos de los lugares paralelos he podido disponer, tanto para ella como para este artículo, de nuevas y meritorias ediciones de casi veinte libros de Livio, posteriores todas a $1974^{3}$. Para la justificación general de mi trabajo me remito a la primera parte de mis "Anotaciones al libro I" y a la introducción de mi edición de ahora. Las siglas utilizadas en estos comentarios son las habituales en las recientes ediciones de la primera década de Livio ${ }^{4}$.

2 A. Fontán, "Anotaciones al texto del libro I de Tito Livio», Emerita 42, 1974, pp. $343-373 ; 43,1975$, pp. 1-24.

${ }_{3}$ Ediciones criticas recientes de Livio, además de la mencionada de Ogilvie, son las siguientes: en la colección Teubner los libros XXI-XXII (ed. Dorey, 1971); XXIII-XXV (ed. Dorey, 1976); XXVI-XXVII, ed. Walsh, 1982; XLI-XLV (ed. Briscoe, 1986). En "Les Belles Lettres", 1. XXXI (ed. Hus, 1976); 1. XXXVI (ed. Manuélian, 1983); 1. XXXVII (ed. Engel, 1983); 1. XXXVIII (ed. Adam, 1982); XLIXLV y fragmentos (ed. Jal, 1971, 1976, 1979). Todas estas ediciones son posteriores a la Concordancia de Packard. En prensa ya este trabajo han aparecido los libros XXVIII-XXX en Teubner (Walsh) y los libros XL (Gouillart) y VIII (Richard) en "Les Belles Lettres".

4 Los pasajes del libro II recientemente discutidos están relacionados en $A N R W$ II 30, 2 (1982) en los trabajos de Kissel (bibliografia general hasta 1978) y Jane E. Phillips (trabajos sobre la primera década, hasta 1979). Para la enumeración de los pasajes y la relación de los trabajos en que se estudian, cf. en ese volumen, p. 951 ss. (especialmente 953 s.), 970 y (para el trabajo critico de Phillips) 1006-1008. Comentarios críticos posteriores en $A P h$ y en los periódicos suplementos bibliográficos de Gnomon.

En este articulo menciono de modo habitual las lecturas de las ediriones de Con- 
1,6 Dissipatae res... forent, quas fouit... moderatio imperii eoque nutriendo perduxit ut bonam frugem... ferre possent.

Possent Ald.: posset N.

La corrección possent de la primera edición Aldina (1518) se habría hallado también en uno de los mss. tardios de Drakenborch, el «Vossianus primus" (cf. Drak. I, p. 240). Este es un códice tardío que contiene la primera y la tercera décadas (cf. Drak. VII, p. $325 \mathrm{~s}$.); también aparece en $F^{3}$ (cf. Conw. Walt. I, ad loc. y p. XVIII). Después de la Aldina, repiten el plural la mayoria de las ediciones. La coincidencia entre los dos mss. sería en todo caso obra del azar, debida a una semejante lectura de dissipatae res como sujeto regular del verbo final de la frase. Weissenborn (hasta la 7. ${ }^{a}$ ed., cuidada ésta por H. J. Müller) había restablecido posset, entendiendo que el verdadero sujeto sería la plebs (ib. $\S 4$ ) de la que se viene hablando desde ocho o diez lineas antes. Ogilvie (A Comm., pp. 235 y 435) siguiendo a Petterson (1930) defiende el sg., que es la forma que luego recoge en su edición, si bien es el único de los editores modernos que lo hace. En apoyo del sg. se aduce un peculiar uso, propio de Tito Livio, de la $3 .^{a} \mathrm{p}$. de possum, acompañada de inf. act. y con valor impersonal. Los lugares paralelos serían otros siete, o sea, ocho en total. Pero yo encuentro una fácil interpretación de varios de ellos, sin necesidad de cambiar el textus receptus, o con las minimas y razonables conjeturas de otros editores. Ocho textos sólo, y discutibles además, en todo Livio, donde hay más de mil empleos de possum en $30^{\mathrm{a}} \mathrm{p}$., son escasos para justificar nada menos que una peculiaridad sintáctica individual de autor, tan llamativa como el uso impersonal de las formas potest, etc., con el valor de «ser posible».

1,11 Conscriptos uidelicet nouum senatum appellabant lectos. Nouum senatum $\mathrm{N}$ : del. ut glossema edd. recc.

Al buen oído latino de Madvig no le sonaba mal el texto tradicional, ni uidelicet significando "a saber" en un pasaje como éste. Weiss.Müll. lo mantienen y lo justifican. El adverbio aparece once veces en

way (OCT, libros I-V), a cuyo prefacio me refiero con los nombres de los dos autores, Conway y Walters. Esta edición ha sido reemplazada más tarde por la de Ogilvie (pero con menos amplia información de variantes); Bayet (ap. "Les Belles Lettres", I. II); Weissenborn-H. J. Müller (utilizada la 11." edición, 1963, que reproduce sin alteraciones la 9.“ de 1908). También menciono frecuentemente los volúmenes I y VII (libros I-IV y comentarios críticos respectivamente) de la edición de Drakenborch (Amsterdam 1738 y 1746) que, como es sabido, reúne una inmensa summa de los comentarios criticos y lecturas anteriores, desde la ed. princeps hasta ésa. Para más amplias referencias bibliográficas me remito a mi edición de la "Colección Hispánica». 
Livio, alguna de las cuales, según H. J. Müller, abonaría la conservación. Los editores del Renacimiento solían añadir in, antepuesto a nouum, lo cual no deja de ser una lectio facilior. Los contemporáneos prefieren suprimir el sintagma n.s., como ya había propuesto Novak. Yo me he inclinado por conservarlo, apoyándome en la tradición, en Madvig y Weissenborn-Müller. Ogilvie (A Comm., ad loc.) defiende la atétesis. Los presuntos lugares paralelos son traídos por unos y otros a favor de sus respectivas posiciones.

2,2 Ac nescio an nimis undique eam minimis quoque rebus muniendo modum excesserint.

Minimis quoque rebus $\mathrm{OH}$ edd. uet. (Drak.): minimisque rebus $\mathrm{M} \lambda$ (praeter $\mathrm{H}$ ).

A favor de minimis quoque rebus se hallan, además de testimonios aislados e independientes de mss. de los grupos $\pi$ y $\lambda$ y el buen sentido latino de los editores antiguos, los textos siguientes: $n e$ in paruis quoque rebus (IV 8,6); diuinis quoque rebus $(\mathrm{X} 8,4)$ y adeo minimis etiam rebus praua religio inserit deos. Nimis... muniendo es igual que nimis detegendo (XXIII 5, 12) y nimis instando (II 64, 7).

\section{2,4 Sermo per totam ciuitatem est datus.}

Datus $\mathrm{N}$ edd.: satus Ogilvie: alii aliter.

El texto es insatisfactorio, tanto el de la tradición, que fue pacificamente aceptada hasta Ruperti, como la ingeniosa conjetura de Ogilvie: porque sermonem o sermones seri es una cosa muy distinta de "extenderse un rumor». Es más bien "empalmar conversaciones». No me he atrevido a proponer ortus que, de ordinario, en Livio acompaña a clamor, fremitus, tumultus, es decir a expresiones de estruendo. Pero también hay en este autor un ortus rumor, menos ruidoso y parecido al sermo, «rumor», «murmuración»: cf. XXVIII 25, 1 y su contexto; y, sobre todo, un sermo... inter tribunos militum secretus oritur (XXXVII 17, 8). $\mathrm{Si}$ yo hubiera reparado antes en este último pasaje, quizá me hubiera decidido por ortus, que es la lectura que ahora recomiendo.

\section{3,2 Nec hi tenui loco orti.}

$H i \mathrm{~N}$ : ii edd., codd. recc.

Creo que el déctico para referirse a los jóvenes recién aludidos es correcto y subraya el carácter nobiliario de las familias de los muchachos, que es lo que Livio quiere destacar aquí. 
4,2 Praeterea aliqui et nobiles adulescentes.

Aliqui et ego: aliquot nobilis $\mathrm{N}$ edd. recc.: aliquot et nobiles Bayet: et aliquot nobiles edd. alii.

El texto que propongo significa, en principio, un número menor de traidores entre los jóvenes de la nobleza que aliquot: «algunos, también nobles...», igual que los Vitelios, los Aquilios y los hijos de Bruto.

4,5 Nam cum pridie quam... proficiscerentur et cenatum forte... esset, coniuratique ibi... egissent.

Et cenatum $\mathrm{N}$ edd. uet. ante et post Dukerum: cenatum Duker, edd. recc.

La necesidad de la atétesis de un et incómodo, porque da lugar a la secuencia et... -que, o su conservación, depende de que se considere ese polisindeton imposible (Ogilvie, $A$ Comm., p. 244) o infrecuente (Weiss.Müll. ad loc.; Drak. id.). En una narración estilísticamente paratáctica y en la que cada frase añade un punto de emoción y de intriga al relato, la serie, ciertamente inusual, et... -que daría al texto una morosidad muy expresiva. Drakenborch decía que la presencia de et traba la frase, que sin la conjunción sería más fluida. Pero no es de desechar que el autor haya intentado precisamente eso.

5,1 De bonis regis.

Regis N: regiis Gruter.

Las dos expresiones significan lo mismo. Me ha parecido preferible seguir la tradición manuscrita. El orden de las palabras, con el genitivo pospuesto, no es un obstáculo en el contexto.

5,4 Vt tam eminens area firma quoque templis ac porticibus sustinendis esset. Firma Duker (cf. Drak. I, p. 255) Ogilvie ego: firmaque $\mathrm{N}$ edd. Quoque templis ego: templis quoque $\mathrm{N}$ edd.

La inversión q.t. de la lectura habitual facilita la atétesis de -que y ofrece un texto más expresivo: la zona, que ya constituia un emplazamiento elevado, alcanzó, gracias a la obra añadida, la solidez precisa para las construcciones grandiosas y suntuarias que en ella se alzarian.

5,10 Post illum obseruatum ut qui ita liberati essent.

Obseruatum N: obseruatum est $\lambda$ (praeter $\mathrm{H}$ ): hoc seruatum Ogilvie.

En efecto, algunos editores renacentistas (cf. Ogilvie, A Comm., p. 247) quieren leer seruatum. Pero Beatus Rhenanus prefiere el obserua- 
tum de la tradición. La argumentación de antiguos y modernos es que obseruatum, en el sentido de guardar una costumbre, resulta duro. Pero Livio emplea este verbo con lex (VI 21,$4 ; 22,10$ ), edictum (tres veces), praecepta, en contextos relativamente parecidos a otros en los que aparece el verbo seruare.

6,2 Ne ex se ortum, eiusdem sanguinis, extorrem, egentem.

Ne ex se ortum... coni. Drak. Conway: ne se ortum eiusdem sanguinis... N: ne se ortum ex Etruscis... Weinkauff Ogilvie: alii aliter.

El texto de $N$ es imposible. Los más significativos editores han querido enmendarlo. La adición de ex Etruscis, es, desde Sigonius que la inventó, el traslado de II 9, 1 (se oriundos ex Etruscis, eiusdem sanguinis nominisque, egentem), que son los términos con que Tarquinio se dirigirá en ese lugar a Porsena. Pero allí la expresión no está inmediatamente precedida de un circumire Etruriae urbes como en 6,2. La razonable objeción de Ogilvie de que se debe referirse a Tarquinio, sujeto de los infinitivos históricos circumire y orare, queda respondida con otra observación del mismo crítico que reconoce que en ante oculos suos perire sinerent hay que aceptar que las miradas que contemplarian la ruina de los Tarquinios serian las de los Etruscos. Se y suos se hallan dentro de la completiva $n e . .$. sinerent, encerrados entre la conjunción y el verbo, de modo que ambos, pronombre y adjetivo posesivo, han de referirse a las mismas personas que evidentemente son los Etruscos en general, y no los Tarquinios, imposibles espectadores de una perdición que les aniquilaba.

7,2 Vocem creditam esse. Haec dicta.

Haec dicta $\mathrm{N}$ edd.: del. Döring Ruperti Ogilvie.

Los enmendantes y el editor británico consideran que es una interpolación. Pero no me parece que haya nada que objetar a la repetición, a corta distancia, de una expresión que en 7,2 es anafórica y en el otro lugar catafórica, si bien este empleo es el más frecuente de h.d. en Livio.

7.6 Ibi alto atque munito loco arcem inexpugnabilem fore.

Fore Weiss.-Müll. Drak. edd. uet.: fieri fore $\mathrm{N}$ : fieri alii edd. recc.

Es preferible fore, porque se refiere al futuro. Como se ve por la facilidad con que se bajan al pie de la colina los materiales, según Livio se trataba más de unos preparativos que de una construcción en marcha. Los usos de fieri y de fore en Livio (cf. Packard) son casi intercam- 
biables y el infinitivo fieri no se refiere nunca a una edificación material.

7,12 Vbi nunc Vicae Potae est.

Vicae Potae Lipsius: uice poce (-ae) N: aedes post Potae add. Siesbye H. J. Müller Ogilvie.

Comenta Ogilvie (A Comm., p. 252) que la elipsis de aedes sólo se encuentra en Vitruvio. ¿Y por qué no aquí, donde acaba de emplearse la forma aedes reiteradamente en otro sentido? Cualquier romano entendería la expresión sin echar nada en falta.

8.2 Bruto statim Horatium suggerunt, credo, quia nulla gesta res insignem fecerit consulatum memoria intercidisse.

Memoria N Frigell Bayet: memoriam Ed. Paris. 1510 H. J. Müller Conway Ogilvie.

A la autoridad de Frigell se une la consideración de que el sujeto del infinitivo es consulatum. Ogilvie ( $A$ Comm., p. 254) decia que tenía que serlo memoriam. El ablativo memoria se halla en Livio con exisset (VI 37, 5), donde $\lambda$ corrige con el acusativo (sic!) y con excessit (VII 32 , 15): en ambos casos sin preposición.

11,2 Ne quid... frumenti subuehi sineret et ut praedatum milites... traicerent. Traicerent N Bayet: traiceret Gronovio Müller Conway Ogilvie.

El sujeto de sineret es Porsenna, pero el de traicerent son los soldados. Hay lugares paralelos en el propio Livio.

12,7 Ibi cum stipendium militibus... daretur et scriba cum rege sedens... multa ageret et eum milites uolgo adirent.

Et eum Schaeffer: eum N Bayet: eumque Ald. Weissenb. Conway Ogilvie.

La pérdida de et se explica por haplografia. Ogilvie ( $A$ Comm., p. 264) parece inclinarse por esta conjetura. Sorprende un poco que, después, en la edición repitiera -que contradiciendo las reservas que él mismo había manifestado respecto de una serie análoga et... -que (cf. supra $4,5)$.

12,12 Rex ira infensus.

Infensus $\mathrm{N}$ edd.: incensus Madvig Ogilvie.

La confusión infensus/incensus (e incluso infestus) es bastante común en contextos emocionales de hostilidad o militares. En contra de infen- 
sus estarian el hecho de que suele llevar el dativo de la persona o realidad contra la que se dirige el ánimo del infensus, y no ablativo, así como el que la palabra habitual para acompañar a ira es incensus o accensus. No obstante, Livio habría escrito infensus ira (inc- Madvig Ogilvie) también en I 53,10 , quo facto... infensus (V 36,11) e infensos... odio (VII 27, 6). Ogilvie corrige el primero de estos dos pasajes, pero mantiene el segundo en su edición, sin hacer ninguna referencia al lugar en $A$ Comm.

12,16 Mea prima sors fuit: ceteri ut cuiusque exciderit primi... aderunt. Vt cuiusque Madvig Conway Ogilvie: utcumque N Weissenb. Bayet. Exciderit Queck (cf. Weiss.-Müll. p. 166 ad loc.): ceciderit $\mathrm{N}$ edd.

Vtcumque obligaba a insatisfactorios equilibrios y a ensayos no logrados de enmienda como el cuique, que se convierte casi en la lectio uulgata desde Nannio (cf. Drak. VII, p. LI) hasta que Madvig introduce la conjetura ut cuiusque, que Conway llamaría brillante.

No es imposible reconstruir el error paleográfico y de sentido que habría conducido desde el $\sim$ que exciderit del arquetipo al -que ceciderit de los códices.

13,2 Mouerat eum et primi periculi casus, quo nihil se praeter errorem insidiatoris texisset.

Quo N: $a$ quo Heumann edd.

Los editores antiguos, que no echaban en falta la preposición, entendian, sin duda, quo, «en el que».

13,8 Sic deditam inuiolatam ad suos remissurum.

Deditam inuiolatam Gruter Drakenb.: deditam inuiolatamque N Rhenanus edd. aliqui: deditam intactam inuiolatamque Froben (1531) edd. uet. et recc. (praeter quosdam ut Drakenborch).

Ogilvie dice ( $A$ Comm., p. 267) que la conservación de -que en $N$ exige un adjetivo previo, y por eso se suma a la inserción de intactam, que proviene del carácter formulario del intactam inuiolatamque de 12 , 14 (cf. también VII 20, 7 y, muy semejante a ambos, VI 3, 10). Pero de esa presunta fórmula, empleada unas líneas antes, procede la enclítica -que, la cual, siguiendo el razonamiento de Ogilvie, se debe atetizar.

15,1 Honoratissimus quisque e patribus.

$E \Delta$ (praeter P): et MP: ex Aldus (edd. post eum) Conway Ogilvie.

$E x$ patribus es más frecuente en Livio (quince lugares); pero $e$ patribus se halla en tres (II 61, 5; IV 4, 7; 23, 4), en el segundo de los cuales 
lo respetan incluso Drakenborch y Ogilvie, que corrigen («dubitanter» el primero de ellos). Es mejor seguir la lectura del arquetipo cuando es posible.

15,3 Eam esse uoluntatem omnium ut qui libertati erit... finis, idem urbi sit. Eam esse uoluntatem edd. uet.: ea $\left(-m \mathrm{M}\right.$ sed del. $\left.\mathrm{M}^{c}\right)$ esse uota eam esse uoluntatem $\mu$ : ea esse uota Hertz Weissenb. Bayet Conway Ogilvie.

Las ditografias de $M$ (y en este caso también del Vormatiense de Rhenanus) son relativamente frecuentes y muy caracteristicas (cf. Ogilvie, $C Q, 1957$, p. 76; $A$ Comm., p. 271). Weissenborn hace observar que con el verbo regente es más adecuado el término uota que uoluntatem. Pero esta palabra no es imposible y está mejor atestiguada.

15,7 Romanis pax fida ita cum Porsenna fuit.

Fida ita $\mathrm{N}$ edd. uet. Weissenb. Bayet: del. ita Madvig Conway: ita Romanis pax fida Weinkauff Ogilvie.

Aunque la posición de ita sea chocante (Ogilvie) y haya invitado a Madvig a la atétesis, propuesta antes por Ussing, la frase recuerda el factum est ita que se encuentra en Cicerón.

18,3 Supra belli Latini metum id quoque accesserat, quod.

Supra belli Latini metum id N: super... Weissenb.: supra belli Latini metum del. Conway Bayet.

Ogilvie ( $A$ Comm., p. 279 ss.) repite sustancialmente el razonamiento de Duker (Drak. I, ad loc.): la guerra Latina a que se tendría miedo es la que se derivaría de la conspiración de los treinta pueblos a que se alude a continuación. Igualmente sigue a Drak. (ib., pero a una nota de éste, que no modificó su texto) para rechazar la construcción supra... metum. Después, el propio crítico inglés, en su edición de 1974, cambió de criterio. Ya no considera preciso sustituir Latini por Sabini, sino que entiende supra como un adverbio y atetiza id. Yo pienso que la interpretación de Doujat (Drak., ib.) es aceptable: al temor de la guerra con los latinos se añadia que ya era conocida la conspiración de los treinta pueblos. La gravedad de la situación aconsejó la creación de la magistratura excepcional del dictator. Respecto de la construcción, yo estimo que los lugares supra modum hominis priuati (IV 13, 3), supra spem humanam (XXXV 14,7) e in fortuna... supra quam ne optare quidem auderet (XLII 15,9) deben ser tenidos en cuenta para mantener el texto del arquetipo. 
21,4 Tanti errores implicantur temporum.

Implicantur ego: implicant N Conway Bayet: errores res implicant Nettleship Ogilvie: implicant tempora Duker (sed non ap. Drak. I ad loc.): implicant temporum rationem Wölfflin Weissenb.

El texto de $N$ es difícilmente inteligible. El verbo implico, para hacer sentido, requeriría un complemento directo: «las grandes diferencias cronológicas" (tanti errores... temporum) confunden o mezclan algo"... ¿a efectos del historiador?... Eso parece sostenerse con la adición de res, paleográficamente justificable por una presunta haplografia. Pero no resuelve el problema: res, "la historia», estaría en singular. Yo pienso que la pasiva encaja bien con el texto y respeta de modo razonable el orden de las palabras. Se trataría, por supuesto, de un uso absoluto, sin ablativo, de la pasiva de implico.

\section{3,14 Frequentique demum curia.}

Demum ego: tandem $\mathrm{N}$ (praeter $\mathrm{U}$ ): inde $\mathrm{U}$, tandem add. s. 1. $\mathrm{U}^{\text {c: }}$ tamen Perizonius: del. Madvig.

La repetición, a cuatro palabras de distancia, del adverbio tandem resulta enojosa para los editores desde Gronovio, que propuso sustituirlo por iam. Weissenb. la atribuye a un descuido; Ogilvie $(A C o m m$., p. $300)$ se afana por buscar lugares paralelos que no acaban de ser satisfactorios. El testimonio de $U$ apunta a una posible vacilación en la tradición. Pero una construcción tandem... demum se encuentra en XXX 21, 9: dedisse id deos tandem sexto decimo demum anno. El adverbio demum cierra con gran frecuencia una serie de enunciados cronológicamente sucesivos.

24,5 Nec posse, cum hostes prope ad portas essent, bello praeuertisse quicquam. Praeuertisse N (per- M ut uid.) edd. uet.: praeuerti se Weissenb.: praeuerti a se Ruperti Pöhlig Bayet: praeuerti Hertz Conway Ogilvie.

Weissenborn plantea una objeción sintáctica. El infinitivo de perf. con posse, afirma, es imposible. Pero en el largo pasaje de estilo indirecto aparecen poco antes interuenisse y unas lineas después cepisse. Ogilvie (AComm., p. 301) insiste en que no hay paralelos para el uso transitivo de praeuertere. Pero, ¿es quicquam necesariamente el sujeto de pos$s e$ ? ¿O éste aquí significa "no era posible»? En ese caso es oportuno recordar otro texto del propio Livio (IX 17, 9). Se halla en el famoso pasaje de Alejandro: deinceps ingentes sequuntur uiri, si Punicum Romano praeuertisset bellum («vienen después grandes héroes en el caso de que hubiera adelantado la guerra contra Cartago a la Romana»). Ade- 
lantar algo a una guerra o una guerra a algo está expresado también con praeuertere (III 23,3 ).

28,3 Nunc in mille curias contionesque, cum alia in Esquiliis, alia in Auentino fiant concilia.

Cum alia in Esquiliis, alia in Auentino fiant concilia N: del. Weinkauff Conway Bayet Ogilvie: alia Esquiliis alia in Auentino Weissenb. Müll., fortasse recte.

No es preciso suponer que se trata de una glosa derivada de 28,1 (coetus nocturnos pars Esquiliis, pars in Auentino facere). En el párrafo 1 es el historiador, o su fuente, quien refiere lo que estaba sucediendo. Aquí, en medio del tumulto, son los "halcones» del Senado los que hablan contra los cónsules y, para ellos, la dispersión topográfica de las conspiraciones es un argumento más para considerar que la situación era particularmente grave. Los editores que suprimen in ante Esquiliis, y también Ogilvie en su comentario (p. 305), se apoyan en la habitual ausencia de preposición con Esquiliae (que parece remontarse a una oposición entre los que "viven fuera", esquilii o esquilini, y los inquilini, que habitan dentro de la urbe). No obstante, el topónimo en cuestión aparece con preposición (ap. Ov., $F$. VI 601) en la mención de la muerte de Servio Tulio: ipse sub Esquiliis, ubi erat sua regia, caesus, y en Tácito (Ann. XV 40), apud imas Esquilias. Los otros usos de Esquiliae que he encontrado, entre ellos siete en Livio (en -as y en -is) no llevan, en efecto, preposición. Sí, en cambio, los adjetivos Esquilinus (in campo Esquilino, Suet., Tib. 14) y Esquilio (monte sub Esquilio, Ov., F. II 435).

30,1 Multis, ut erat, horrida et atrox uidebatur Appi sententia; rursus Vergini Largique exemplo haud salubres, utique Largi putabant sententiam, quae totam fidem tolleret.

Putabant sententiam N: del. Gebhard (ap. Drakenb. I 364) edd. recc. praeter Ogilvie: add. eam esse Ogilvie.

El pasaje planteaba problemas de interpretación ya a Rhenano, que se preguntaba cómo lo habría explicado Beroaldo en sus lecciones de Bolonia. Es Beatus Rhenanus el que fija la puntuación aquí seguida. Por fin Gebhard propone la atétesis de putabant sententiam poniendo punto antes de Vtique. Ogilvie ( $A$ Comm., p. 306) sugiere, en primer lugar, que haya un punto («strong stop») después de salubres, y luego echa en falta alguna especie de predicado de sententiam, entendida esta voz como sujeto de un esse. (Hay que observar que ya Beatus Rhenanus había advertido que con un eam esse o un talem esse todo seria más fácil.) Pero en el propio Livio creo yo encontrar varios usos de putare no seguido de infinitivo, con su complemento directo acompañado de 
un participio, o incluso de un adjetivo en función ciertamente predicati$\mathrm{va}$, que es la que aquí desempeñaría la oración de quae: omnem neglegentiam diuini cultus exemptam hominibus putem (V 51, 5); quid optimum putarent (XXXII 19, 10); uictos se an uictores putarent (VII 25, 5); quoad tutum putaret (XXVII 39, 3).

\section{1,2 Parum apte introrsum ordinibus aciem firmauerant. Introrsum $\mathrm{N}$ edd.: introrsus $\mathrm{O}$ Ogilvie.}

Las discusiones de los editores y críticos conciernen al modo como, según Livio, se habrian desplegado las tropas y se habrían movido luego. Mi diferencia con Ogilvie, que, a mi juicio, ofrece una explicación convincente de ello, afecta sólo al adverbio. Como se trata de describir una formación estática, para Ogilvie debería ser introrsus y no introrsum, que en Livio (Ogilvie, A Comm., p. 308) sólo se emplearía cuando hay movimiento. Yo no veo que la distribución sea tajante ni en Livio ni en otros autores. He preferido seguir el texto de $N$.

\section{4,11 Fruantur annona quam furore suo fecere.} Fruantur utantur $\mu$ : utantur $\Delta$.

Rhenanus pensó que fruantur, documentado en su códice, podría usarse absolutamente, sin complemento, refiriéndose a un eliptico raptis, implícito en el contexto de los saqueos de la línea precedente.

Parece claro que se trata de una doble lectura del arquetipo de las que en ocasiones se conservan en uno o los dos (como en este caso) códices $\mu$ (Vorm. y $M$ ).

Ninguno de los dos verbos es específico para la cosecha anual de cereales. Fruantur me parece más expresivo, especialmente al lado de la locura (furor) que ha dado lugar a la situación.

\section{6,1 Seruum quidam pater familiae... sub furca caesum medio egerat circo.} Virgis post caesum add. Ogilvie.

Es frecuente, como dice Ogilvie en su comentario, que el participio de caedo esté acompañado de la mención del instrumento con que se azotó a la víctima - uirgis, flagro, etc.-; así ocurre en los lugares de Livio en que caesi, caesa, caesos, etc., no se refieren a los caídos en una batalla. Pero también en otros textos latinos se emplea sin ese ablativo. Hay un pasaje del libro I del propio Livio, cuyo paralelismo con éste es manifiesto: sub furca uinctum (Horacio en I 26, 10) y sub furca caesum aquí el esclavo en cuestión. 
38,2 Ibi in contionis modum orationem exorsus, "Vt ueteres populi Romani iniurias cladesque gentis Volscorum, ut omnia, inquit, obliuiscamini...".

$V t$ ueteres Drak. Bayet: post exorsus add. exsecutus F. Walters exsecutusque Ogilvie: alii aliter.

Ogilvie llama la atención sobre que inquit exige su emplazamiento al principio de la frase o dentro del primer miembro. Hay algún pasaje del propio Livio en que esto no está tan claro: quod cum ita se habeat, cui deorum hominumue indignum uidere potest, inquit, eos uiros quos... (X 7, 9). La conjetura exsecutus (Walters), ampliada a exsecutusque (Ogilvie), es verdaderamente ingeniosa: significaría "y enumerando las viejas ofensas del pueblo romano...». Pero no parece necesaria la inserción del participio.

38,4 Quid eos qui audiuere uocem praeconis, qui nos uidere abeuntes, quid eos qui huic ignominioso agmini fuere obuii.

Post nos add. eos Hearn (cf. Drak. I, p. 409) Ogilvie.

La adición quiere restablecer un riguroso paralelismo en la construcción trimembre mediante la repetición de quid eos qui. La uariatio quid eos qui... quid qui nos... quid eos qui... también tiene su gracia. Sería extraño que en una reiteración del tipo de la propuesta se hubiera perdido el segundo de tres eos en toda la tradición, y siempre el mismo.

41,4 Fastidire munus uolgatum a ciuibus esse in socios.

Esse Vorm. (a quodam lectore scriptum) $\lambda$ (praeter $\mathbf{H}$ ) Weiss.: egisse $\mathbf{M}$ : isse $\pi$ $\mathrm{H}$ : ipsis Ogilvie: esse uel sese Drak. (in annotat.): secl. Gronov.

La argumentación de Ogilvie sobre la historia de la tradición le obliga a postular un doble esse (probablemente isse en $N$ ); y luego el análisis del texto a que munus uolgatum sea complemento directo de fastidire. Si es seguro el se inspici, aestimari fastidiat (VI 41,2), ¿por qué no aquí uolgatum esse? El tiempo pasado es correcto, porque la decisión de extender el reparto de tierras a los aliados latinos era ya un hecho.

41,6 Quid ita enim adsumi socios et nomen Latinum, quid attinuisset Hernicis, paulo ante hostibus, capti agri partem tertiam reddi...?

Attinuisset N Conway Bayet: attinuisse edd. uet. post Aldum et Rhenanum, Weiss. Ogilvie.

La argumentación de Ogilvie ( $A$ Comm., p. 341 s.) a favor de una secuencia de tres infinitivos, con el tercero dependiente del segundo, no es concluyente. Son dos operaciones politicas distintas, la de los latinos, a los que se asignaba un tercio del territorio de los hérnicos, y la de de- 
volver a estos últimos otro tercio. Ambas dependen de attinuisset, en subjuntivo por el estilo indirecto.

41,9 Adeo propter suspicionem insitam regni... munera eius animis hominum respuebantur.

Animis hominum Gaertn. (ap. Drak.): in animis hominum $\mathrm{N}$ edd. uet.: animis hominum Weiss.: in animis hominum Alan Ogilvie transposuerunt ante insitam: in animis hominum secl. Conway, Vielhaber secutus qui et insitam delebat.

Ogilvie dice, con razón, que in animis hominum entre eius y respuebantur no es latín, pero el ablativo agente animis hominum sí. In sería uno de esos monosílabos intrusos de que habla Havet, que no se reducen a conjunciones, sino que también son preposiciones. Se trata de una tosca lectio facilior.

46,1 Prope certa spes erat non magis secum pugnaturos quam pugnarint cum Aequis. Pugnarint M: pugnauerint $\Delta$ (praeter $\mathrm{H}$ ) edd. Weiss. Ogilvie: om. H Conway Bayet.

Las formas sincopadas, dice H. J. Müller (p. 174), son en Livio menos frecuentes que las plenas. No obstante, en este mismo libro se hallan uiolarit $(29,12)$, maturarimus $(38,5)$; el propio critico alemán cita otros casos de formas sincopadas de perfecto de subjuntivo o futuro segundo de la primera conjugación en libros ajenos a la década, por lo que se ve que no es una peculiaridad de los escribas o del modelo de $M$.

\section{7,11 Adeo spreta in tempore gloria interdum cumulatior rediit.} Rediit $\mathrm{N}$ edd.: redit Aldus Ogilvie.

Parece, ciertamente, un proverbio, como afirma Ogilvie (A Comm., p. 358). Pero el perfecto no es obstáculo para ello.

48,6 Moturos apparebat Sabinos semper infestos Etruriamque omnem.

Post moturos add. se $\mathrm{N}$ edd. uet.: secl. Madvig Conway: esse Seyffert Weiss. Bayet Ogilvie.

El auxiliar es innecesario; pero además no se halla en ninguno de los otros siete pasajes del autor en que se encuentra moturos.

50,6 Et cum improuidi effuso cursu insidias circa ipsum iter locatas superassent. Insidias $\mathrm{N}$ edd.: praesidia... locata Ogilvie: custodias uel praesidia Ogilvie ( $A$ Comm., p. 365).

Tal como lo cuenta $N$ la tropa romana de los Fabios rebasó a los adversarios que se habian apostado en torno al camino, sin darse cuen- 
ta de su presencia. Cuando luego los Fabios se dispersan para perseguir unos rebaños, se encuentran enfrente con unos enemigos, a cuya acción se une la de los que estaban ocultos.

Haciendo caso de la conjetura de Ogilvie no se sabía de qué escondrijo - cf. infra, ex insidiis - habrian salido los Etruscos que envolvieron a los Fabios.

\section{0,11 Vnum prope impuberem aetatem relictum.}

Prope impuberem aetatem ego: propter impuberem aetatem Kreyssig (cf. Aur. V., de uir. ill. 14, 6) Madvig Ogilvie: prope puberem aetatem M: prope puberem aetate $\Delta$ edd. Weiss. Conway Bayet.

Prope puberem (impuberem) aetatem tiene el apoyo de dos pasajes del libro I: imperium ei (Ascanio) ad puberem aetatem incolume mansit $(3,1)$; iam filii (Anci regis) prope puberem aetatem erant $(35,1)$. Prope, además, se emplea en Livio no pocas veces como preposición no sólo en contextos de lugar, sino de tiempo o similares y junto a conceptos abstractos: prope maturitatem (segetum) (XXVIII 5, 15); prope quadringentesimum annum (V 45, 4); prope metum (I 25, 13); prope perniciem (XXI 34, 8). Desde luego en todas las épocas de la latinidad prope es más aplicado al espacio que al tiempo, pero incluso la forma propius en uso preposicional se usa en contextos no espaciales (aunque en Hofmann-Szantyr, Lat. Gramm. II, p. 245, se diga "nur in lokaler Bedeutung»). Cf. en Livio propius periculum fuerint (XXI 1, 2); propius fastidium sui quam desiderium (XXVIII 40, 9). En resumen, que, a mi juicio, el De uiris suministra impuberem, pero no necesariamente propter.

\section{2,5 Inde morbo absumptum.}

Absumptum M Conway Bayet; add. esse $\Delta($ est $\mathrm{O})$ edd. Weiss. Ogilvie.

Ogilvie dice que hay que conservar el verbo auxiliar, sin aportar razones. Pero el aire rápido, entrecortado y casi lapidario del pasaje, recomienda, a mi juicio, lo contrario. A lo cual se une la autoridad de $M$ y la relativa abundancia de infinitivos pasivos sin esse en Livio.

54,4 Consulares uero fasces, praetextam, curulemque sellam nihil aliud quam pompam funeris putent.

Post praetextam add. togam Ogilvie.

En otros lugares, en efecto, Livio emplea toga praetexta, e incluso junto a curuli sella o sella curulis (I 8, 3; X 7, 9; XXVII 8, 9; XXX 11, 12 ), si bien en el del libro $X$ se enumeran varias clases de togas, una de 
las cuales es la praetexta. En ningún lugar, sin embargo, praetexta toga y sí praetextam sólo (XXI 63,10) como en otros numerosos autores.

\section{6,2 Rogationem tulit ad populum ut plebei magistratus tributis comitiis fierent. Plebei M $\lambda$ U: plebeii O edd. Ogilvie: plebi et $\pi$ (praeter U).}

Hay otro pasaje (IV 25,11$)$ en que parece que los mss. están contestes en plebeii (habría que revisarlos), porque plebei puede ser, como en este lugar, un genitivo, apoyado por la autoridad de la tradición y por la similitud de expresiones como aedilis, tribuni plebei, si bien no se puede dejar de reconocer que aqui el orden de las palabras está invertido.

\section{6,6 Vt inuentor legis Volero, sic Laetorius, collega eius, auctor... erat.} Ante Laetorius add. C. Münzer (cf. D. H. IX 46, 1) Ogilvie.

Münzer ( $a p . R E$ ) señala que el praenomen pudo haber caído por haplografia. En efecto, como también dice Ogilvie, Tito Livio gusta de presentar a los personajes nuevos con cierta formalidad, incluyendo el praenomen. Pero éste, ciertamente, no está en los manuscritos y, además, aquí lo que quiere destacar el autor es el contraste (inuentor... auctor) y la similitud collega eius de Letorio con Volerón, que tampoco presenta praenomen.

56,12 Facile et contemptim de iure disserendo perturbare Laetorium poterat. Et contemptim $\mathrm{N}$ edd. Bayet: del. et Drak. Weiss. Conway Ogilvie.

Dos adverbios unidos por una conjunción son una construcción normal y aceptable. El razonamiento de Drakenborch es indirecto (I, p. 499) y no llega a una conclusión definitiva.

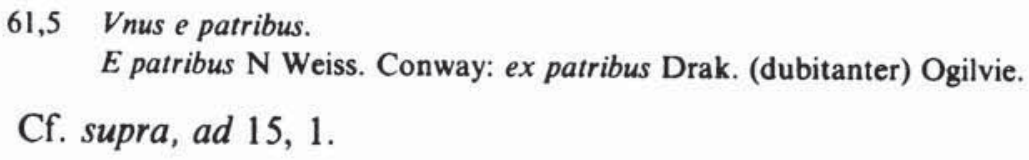

Antonio Fontán 